\title{
WEIGHTED NORM INEQUALITIES FOR THE CONJUGATE FUNCTION AND HILBERT TRANSFORM
}

\author{
BY
}

\section{RICHARD HUNT(1), BENJAMIN MUCKENHOUPT(2) AND RICHARD WHEEDEN(3)}

ABSTRACT. The principal problem considered is the determination of all nonnegative functions $W(x)$ with period $2 \pi$ such that

$$
\int_{-\pi}^{\pi}|\tilde{f}(\theta)|^{p} \mathbb{W}(\theta) d \theta \leq C \int_{-\pi}^{\pi}|f(\theta)|^{p} W(\theta) d \theta
$$

where $1<p<\infty, f$ has period $2 \pi, C$ is a constant independent of $f$, and $\tilde{f}$ is the conjugate function defined by

$$
\tilde{f}(\theta)=\lim _{\epsilon \rightarrow 0^{+}} \frac{1}{\pi} \int_{\epsilon \leq|\phi| \leq \pi} \frac{f(\theta-\phi) d \phi}{2 \tan \phi / 2} .
$$

The main result is that $W(x)$ is such a function if and only if

$$
\left[\frac{1}{|I|} \int_{I} \mathbb{W}(\theta) d \theta\right]\left[\frac{1}{|I|} \int_{I}[\mathbb{W}(\theta)]^{-1 /(p-1)} d \theta\right]^{p-1} \leq K
$$

where $I$ is any interval, $|I|$ denotes the length of $I$ and $K$ is a constant independent of $I$.

Various related problems are also considered. These include weak type results, the nonperiodic case, the discrete case, an application to weighted mean convergence of Fourier series, and an estimate for one of the functions in the Fefferman and Stein decomposition of functions of bounded mean oscillation.

1. Introduction. The natural weighted norm inequality problem for the conjugate function is the characterization of those finite nonnegative Borel measures $m$ on $[-\pi, \pi]$ for which

$$
\left[\int_{-\pi}^{\pi}|\tilde{f}(\theta)|^{p} d m(\theta)\right]^{1 / p} \leq C\left[\int_{-\pi}^{\pi}|f(\theta)|^{p} d m(\theta)\right]^{1 / p}
$$

for a given $p, 1<p<\infty$, with $C$ independent of $f$. In order for such a norm inequality to hold, however, it is necessary that $m$ be absolutely continuous with respect to Lebesgue measure; see [7] and [4, p. 373]. Thus we may express

Presented to the Society, January 19, 1972; received by the editors January 18, 1972. AMS (MOS) subject classifications (1969). Primary 4430; Secondary 2649.

Key words and phrases. Conjugate function, Hilbert transform, discrete Hilbert transform, weighted norm inequalities, bounded mean oscillation.

(1) Supported in part by NSF grant GP-18831 and the Alfred P. Sloan foundation.

(2) Supported in part by NSF grant GP-20147.

(3) Supported in part by NSF grant GP-20132. 
$d m(\theta)=W(\theta) d \theta$ where $W$ is non negative and rewrite (1.1) as

$$
\left[\int_{-\pi}^{\pi}|\tilde{f}(\theta)|^{p} W(\theta) d \theta\right]^{1 / p} \leq C\left[\int_{-\pi}^{\pi}|f(\theta)|^{p} W(\theta) d \theta\right]^{1 / p} .
$$

The problem of characterizing those weight functions $W$ for which (1.2) holds with $p=2$ was solved by $\mathrm{H}$. Helson and G. Szego in [7]. The ir remarkable result is that $W$ satisfies (1.2) with $p=2$ if and only if $W(\theta)=\exp (U(\theta)+\widetilde{V}(\theta))$ where $U$ and $V$ are bounded functions and the strict inequality $\|V\|_{\infty}<\pi / 2$ holds. It is a relatively simple matter to prove the sufficiency of this condition by adapting the classical M. Riesz proof of the $L^{2}$ boundedness of the conjugate function; see Lemma 6 below and [5]. The proof of the necessity is more difficult and is based in part on a duality argument for the Hardy space $H^{1}$.

For $p \neq 2$ Forelli in [4] obtained a necessary and sufficient condition for $W$ to satisfy (1.2) but the condition is difficult to apply. Other sufficient conditions have been given in [1], [4], [5], and [12].

Our solution to the problem has several interesting features. Our condition is simple to state, it is a necessary and sufficient condition for both strong and weak type inequalities, and it is the same as the condition for norm inequalities of the Hardy-Littlew ood maximal function as proved in [9].

To state our main result we will use the following notation. For an interval $I,|I|$ is the length of $I$. For functions $f$ with period $2 \pi$,

$$
T f(\theta)=\sup _{\epsilon>0}\left|\frac{1}{\pi} \int_{\epsilon \leq|\phi| \leq \pi} \frac{f(\theta-\phi) d \phi}{2 \tan \phi / 2}\right|
$$

$D_{a}$ is the subset of $[-\pi, \pi]$ where $|\tilde{f}(\theta)|>a$ and $E_{a}$ is the subset of $[-\pi, \pi]$ where $T f(\theta)>a$. For $p=1$,

$$
\left(\int_{I}[g(\theta)]^{-1 /(p-1)} d \theta\right)^{p-1}
$$

will be taken to mean ess $\sup _{\theta \in I} 1 /|g(\theta)|$, and $0 \cdot \infty$ will be taken to be 0 . To avoid complications in one pathological case, $\tilde{f}(\theta)$ and $T f(\theta)$ will be assigned the value $\infty$ if $f$ is not integrable. The theorem we will prove in the periodic case is the following.

Theorem 1. If $W(\theta)$ is nonnegative and has period $2 \pi$ and $1<p<\infty$, then the following statements are equivalent; if $p=1$ statements (a), (d), and (e) are equivalent.

(a) There is a constant $C$, independent of $I$, such that for every interval $I$,

$$
\left[\frac{1}{|I|} \int_{I} W(\theta) d \theta\right]\left[\frac{1}{|I|} \int_{I}[W(\theta)]^{-1 /(p-1)} d \theta\right]^{p-1} \leq C .
$$

(b) There is a constant $C$, independent of $f$, sucb that for every $f$ of period $2 \pi$ 


$$
\int_{-\pi}^{\pi}|\tilde{f}(\theta)|^{p} W(\theta) d \theta \leq C \int_{-\pi}^{\pi}|f(\theta)|^{p} W(\theta) d \theta .
$$

(c) There is a constant $C$, independent of $f$, such that for every $f$ of period $2 \pi$

$$
\int_{-\pi}^{\pi}[T f(\theta)]^{p} W(\theta) d \theta \leq C \int_{-\pi}^{\pi}|f(\theta)|^{p} W(\theta) d \theta .
$$

(d) There is a constant $C$, independent of $f$ and a, such that for every $f$ of period $2 \pi$ and every $a>0$,

$$
\int_{D_{a}} W(\theta) d \theta \leq C a^{-p} \int_{-\pi}^{\pi}|f(\theta)|^{p} W(\theta) d \theta
$$

(e) There is a constant $C$, independent of $f$ and $a$, such that for every $f$ of period $2 \pi$ and every $a>0$,

$$
\int_{E_{a}} W(\theta) d \theta \leq C a^{-p} \int_{-\pi}^{\pi}|f(\theta)|^{p} W(\theta) d \theta
$$

Condition (a) in Theorem 1 was used in an equivalent form by Rosenblum in [14] to characterize weight functions which give norm inequalities for the Poisson integral. It was also used in [9] to characterize weight functions for the HardyLittlewood maximal function.

It should be noted that if $W(\theta)$ satisfies statement (a) and for some $I, \int_{I} W(\theta) d \theta=\infty$, then $\int_{J}[W(\theta)]^{-1 /(p-1)} d \theta=0$ for every interval $J$. Therefore, $W(\theta)=\infty$ for almost every $\theta$. Similarly, if $\int_{I}[W(\theta)]^{-1 /(p-1)} d \theta=\infty$ for some $I$, then $W(\theta)=0$ for almost every $\theta$. Except for the se two pathological cases a $W$ that satisfies statement (a) satisfies $0<W(\theta)<\infty$ almost everywhere and $W(\theta)$ and $[W(\theta)]^{-1 /(p-1)}$ are integrable on $[-\pi, \pi]$.

An application of Theorem 1 to mean convergence of Fourier series is given in \$6. Results like Theorem 1 are true for the Hilbert transform and the discrete Hilbert transform. The exact statements and sketches of their proofs are given in $\$ \$ 7$ and 8.

The various equivalences in Theorem 1 are particularly interesting for $p=2$ for then statement (a) has a particularly simple form and expresses a condition equivalent to the one given by Helson and Szego. There are other interesting formulations in this case. To state the se the notation

$$
P(g, r, \theta)=\frac{1}{2 \pi} \int_{-\pi}^{\pi} \frac{\left(1-r^{2}\right) g(\theta-\phi) d \phi}{1-2 r \cos \phi+r^{2}}
$$

will be used for the Poisson integral. These formulations are as follows.

Theorem 2. If $W(\theta)$ is nonnegative and has period $2 \pi$, then the following statements are equivalent.

(a) There is a constant $C$, independent of $I$, such that for every interval $I$,

$$
\left[\frac{1}{|I|} \int_{I} W(\theta) d \theta\right]\left[\frac{1}{|I|} \int_{I} \frac{d \theta}{W(\theta)}\right] \leq C
$$


(b) There are bounded functions $U(\theta)$ and $V(\theta)$ of period $2 \pi$ with $\|V(\theta)\|_{\infty}<$ $\pi / 2$ such that $W(\theta)=\exp [U(\theta)+\widetilde{V}(\theta)]$ or $W(\theta)=0$ almost everywhere or $W(\theta)=\infty$ almost everywhere.

(c) There is a constant $C$, independent of $r$ and $\theta$, sucb that for all $\theta$ and $0 \leq r<1, P(W, r, \theta) P(1 / W, r, \theta) \leq C$.

(d) There is a constant $C$, such that for all $\theta$ and $0 \leq r<1, P(W, r, \theta) \leq$ $C \exp [P(\log W, r, \theta)]$ and $P(1 / W, r, \theta) \leq C \exp [P(-\log W, r, \theta)]$.

(e) There is a constant $C$, independent of $I$, sucb that for all intervals $I$,

$$
\frac{1}{|I|} \int_{I} \exp \left[\left|\log W(\theta)-D_{I}\right|\right] d \theta \leq C
$$

for some $D_{I}$ depending on $I$ or $W(\theta)=0$ almost everywbere or $W(\theta)=\infty$ almost everywhere.

Another interesting consequence of Theorem 1 is that it gives information concerning the size of one of the functions in the Fefferman and Stein decomposition of functions of bounded mean oscillation.

Theorem 3. Assume that $f(\theta)$ bas period $2 \pi$ and is of bounded mean oscillation. Let $A$ be the least upper bound of all real numbers, a, such that $W(\theta)=$ $\exp a f(\theta)$ satisfies statement (a) of Theorem 2. Let $B$ be the greatest lower bound of all $b$ such that $f(\theta)$ can be written in the form $U(\theta)+\widetilde{V}(\theta)$ with $U(\theta)$ bounded and $|V(\theta)| \leq b$. Then $B=\pi / 2 A$.

Theorems $1-3$ are consolidations of various results in $\$ \$ 2-5$. In $\$ 2$ various properties are proved for functions satisfying the condition in part (a) of Theorem 1 . In $\$ 3$ these results are used to prove that for $p>1$ statement (a) in Theorem 1 implies statements (b) and (c); these implications are called Theorems 4 and 5. Their proofs are based on three main points: the Calderon-Zygmund decomposition; the fact from [9] that if $W$ satisfies statement (a) for a $p>1$ it also satisfies it for a smaller value of $p$; and finally results from [3] characterizing functions of bounded mean oscillation.

In Theorem 1 the implications $(c) \Rightarrow(e),(e) \Rightarrow(d)$, and $(b) \Rightarrow(d)$ are trivial. Therefore, the proof of Theorem 1 for $p>1$ can be completed by proving that $(d) \Rightarrow(a)$. This is relatively simple and is done in $\$ 5$. For $p=1$ Theorem 1 is proved by showing that (a) implies (e) and (d) implies (a) since (e) trivially implies (d). The proof that (a) implies (e) is given in $\$ 4$ while the proof that (e) implies (a) is in $\$ 5$.

Theorem 2 is largely a by-product of the proof of Theorem 1. The equivalence of parts (a) and (b) follows from the equivalence of part (a) with part (b) of Theorem 1 for $p=2$ and the Helson-Szego result mentioned above. Lemma 3 shows that (a) implies (c) while the converse is trivial. Lemma 4 is the proof 
that (a) is equivalent to (e). Part (c) implies part (d) by use of the fact, obtained from Jensen's inequality, that the opposite inequalities in (d) are true for any function. Part (d) implies (c) trivially.

Theorem 3 is a simple consequence of Theorem 2. If $A>0$ then given $a$ satisfying $0<a<A$ there is an $a_{1}>a$ such that $\exp a_{1} f(\theta)$ satisfies statement (a) of Theorem 2. By statement (b) there are bounded functions $U(\theta)$ and $V(\theta)$ such that $\|V(\theta)\|_{\infty}<\pi / 2$ and $a_{1} f(\theta)=U(\theta)+\widetilde{V}(\theta)$ for almost all $\theta$. Then $B \leq$ $\pi / 2 a_{1}<\pi / 2 a$, and it follows that $B \leq \pi / 2 A$. This is also true for $A=0$. Conversely, if $B<\infty$, given $b>B$ there are bounded functions $U(\theta)$ and $V(\theta)$ such that $f(\theta)=U(\theta)+\widetilde{V}(\theta)$ and $\|V(\theta)\|_{\infty}<b$. Then $\pi f(\theta) / 2 b$ satisfies statement (b) of Theorem 2. Since it also must satisfy statement (a), $\pi / 2 b \leq A$. It follows that $\pi / 2 B \leq A$; this is also true for $B=\infty$. This completes the proof of Theorem 3 .

Throughout this paper $C$ will denote a constant not necessarily the same at each occurrence.

2. Facts about the $A_{p}$ condition. A function $W(x)$ will be said to satisfy the $A_{p}$ condition with constant $C$, if $W(x)$ is nonnegative, $0<W(x)<\infty$ on a set of positive measure, $C<\infty$ and for every interval $I$,

$$
\left[\frac{1}{|I|} \int_{I} W(x) d x\right]\left[\frac{1}{|I|} \int_{I}[W(x)]^{-1 /(p-1)} d x\right]^{p-1} \leq C .
$$

For $p=1$ the convention stated before Theorem 1 makes (2.1) equivalent to the statement $W^{*}(x) \leq C W(x)$ where

$$
W^{*}(x)=\sup _{\substack{y \neq x \\ y-x}} \int_{x}^{y} W(t) d t
$$

is the Hardy-Littlewood maximal function of $W(x)$.

Two simple observations about the $A_{p}$ condition will be used repeatedly. If $W(x)$ satisfies the condition $A_{p}$ with constant $C$ for some $p \geq 1$, then for every $r>p$ Hölder's inequality shows that $W(x)$ satisfies condition $A_{r}$ with constant $C$. It also follows directly from the definition that if $W(x)$ satisfies the $A_{p}$ condition with constant $C$ for some $p>1$, then $[W(x)]^{-1 /(p-1)}$ satisfies the condition $A_{p^{\prime}}$ with constant $C^{1 /(p-1)}$ where $1 / p+1 / p^{\prime}=1$. The following result from [9] will also be needed.

Lemma $A$. If $1<p<\infty$ and $W(x)$ satisfies condition $A_{p}$ with constant $C$, then there are constants $r$ and $K$, depending only on $p$ and $C$, such that $1<r<p$ and $W(x)$ satisfies condition $A_{r}$ with constant $K$.

This is essentially Lemma 5 of [9]; for a proof see [9].

The following result of Stein and Fefferman concerning functions of bounded mean oscillation will also be needed. As usual, a function $f(x)$ will be defined 
to be of bounded mean oscillation if there is a constant $K$ such that for every interval $I$,

$$
\frac{1}{|I|} \int_{I}|f(x)-A| d x \leq K
$$

for some $A$ depending on $I$.

Lemma B. If $b(\theta)$ is of bounded mean oscillation and bas period $2 \pi$, then there exist bounded functions $u(\theta), v(\theta)$ with period $2 \pi$ such that $b(\theta)=u(\theta)+$ $\widetilde{v}(\theta)$ for almost every $\theta$.

This result was announced in [2] and proved in [3] for the nonperiodic case. The proof for the periodic case is the same.

The following lemmas will be needed in $\$ 3$ to prove that $A_{p}$ is a sufficient condition for a function to be a weight function. Some have been stated in a stronger form than needed when the result was interesting and the proof was simple.

Lemma 1. If $1<p<\infty$ and $W(x)$ satisfies the $A_{p}$ condition with constant $C$, then there is a constant $K$, depending only on $p$ and $C$, sucb that for every interval $l$,

$$
\int_{x \notin I} \frac{|I|^{p-1} W(x) d x}{\left|x-a_{I}\right|^{p}} \leq \frac{K}{|I|} \int_{I} W(x) d x,
$$

where $a_{I}$ denotes the center of $I$.

By Lemma $A$ there are constants $r$ and $D$, depending only on $p$ and $C$, such that $1<r<p$ and $W(x)$ satisfies condition $A_{r}$ with constant $D$. We will show that (2.3) holds with $K=D p /(p-r)$. Without loss of generality we may take $I$ to have the form $[-b, b]$; it will then be sufficient to show that

$$
\int_{b}^{\infty} x^{-p} W(x) d x \leq \frac{D p b^{-p}}{p-r} \int_{0}^{b} W(x) d x .
$$

Since $W(x)$ satisfies condition $A_{r}$ with constant $D$, for every $y .>0$,

$$
\int_{0}^{y} W(x) d x\left[\int_{0}^{y}[W(x)]^{-1 /(r-1)} d x\right]^{r-1} \leq D y^{r} .
$$

This immediately implies that for $y \geq b$,

$$
y^{-p-1}\left[\int_{b}^{y} W(x) d x\right]\left[\int_{0}^{b}[W(x)]^{-1 /(r-1)} d x\right]^{r-1} \leq D y^{r-p-1} .
$$

By Hölder's inequality applied to $\int_{0}^{b}[W(x)]^{1 / r}[W(x)]^{-1 / r} d x$ we obtain

$$
\left[\int_{0}^{b}[W(x)]^{-1 /(r-1)} d x\right]^{r-1} \geq b^{r}\left[\int_{0}^{b} W(x) d x\right]^{-1} \text {. }
$$

Using this in (2.5) leads to 


$$
y^{-p-1} \int_{b}^{y} W(x) d x \leq D b^{-r} y^{r-p-1} \int_{0}^{b} W(x) d x .
$$

Now integrate (2.6) in $y$ from $b$ to $\infty$. Interchanging the order of integration on the left and performing the integration on the right leads immediately to (2.4).

Lemma 2. If $1 \leq p<\infty, W(x)$ satisfies the $A_{p}$ condition and $\int_{-\infty}^{\infty}|f(x)|^{p} W(x) d x$ $<\infty$, then $\int_{-\infty}^{\infty}|f(x)|(1+|x|)^{-1} d x<\infty$.

By Hölder's inequality if $p>1$, the integral in the conclusion of Lemma 2 is bounded by

$$
\left[\int_{-\infty}^{\infty}|f(x)|^{p} W(x) d x\right]^{1 / p}\left[\int_{-\infty}^{\infty} \frac{[W(x)]^{-1 /(p-1)} d x}{(1+|x|)^{p^{\prime}}}\right]^{1 / p^{\prime}}
$$

The first part of (2.7) is finite by hypothesis. It was observed above that if $W(x)$ satisfies the $A_{p}$ condition then $W(x)^{-1 /(p-1)}$ satisfies the condition $A_{p^{\prime}}$. Then using Lemma 1 shows that the second integral in (2.7) is bounded by a constant times $\int_{-1}^{1}[W(x)]^{-1 /(p-1)} d x$. This last integral is finite since the pathological case that would make it infinite was ruled out in the definition of $A_{p}$. This completes the proof of Lemma 2 for $p>1$. If $p=1$,

$$
W(x) \geq C W^{*}(x) \geq \frac{C}{1+|x|} \int_{-1}^{1} W(y) d y .
$$

Since $\int_{-1}^{\mathrm{i}} W(y) d y>0$, the conclusion also follows in this case.

Lemma 3. If $1 \leq p \leq 2$ and $W(x)$ satisfies the $A_{p}$ condition with constant $C$, then there is a constant $K$, depending only on $p$ and $C$ such that for every interval $I$,

$$
\int_{x \notin I} \frac{|I| W(x) d x}{\left(x-a_{I}\right)^{2}} \leq \frac{K}{|I|} \int_{I} W(x) d x
$$

where $a_{I}$ denotes the center of $I$.

This follows immediately from Lemma 1 since if $W(x)$ satisfies such an $A_{p}$ condition, it also satisfies $A_{2}$.

Lemma 4. $W(x)$ satisfies the $A_{2}$ condition if and only if there is a constant $K$, such that for every interval $I$,

$$
\frac{1}{|I|} \int_{I} \exp [|\log W(x)-\log A|] d x \leq K
$$

for some $A>0$ depending on $I$.

If $W(x)$ satisfies the $A_{2}$ condition with constant $C$, fix $I$ and let $A=$ $|I|^{-1} \int_{I} W(x) d x$. The left side of (2.8) is then bounded by 


$$
\frac{1}{|I|} \int_{I} \frac{W(x) d x}{A}+\frac{1}{|I|} \int_{I} \frac{A d x}{W(x)}
$$

Substituting in the value of $A$ shows that the first term of (2.9) is 1 and by the $A_{2}$ condition the second term is bounded by $C$. This proves (2.8) with $K=C+1$.

If (2.8) is true, fix an $I$ and observe that

$$
\frac{1}{|I|} \int_{I} \frac{W(x) d x}{A}+\frac{1}{|I|} \int_{l} \frac{A d x}{W(x)} \leq 1+\frac{1}{|I|} \int_{I} \exp [|\log W(x)-\log A|] d x,
$$

and the right side of (2.10) is bounded by $K+1$ for some $A$. The value of $A$ that minimizes the left side of $(2.10)$ is

$$
\left[\int_{I} W(x) d x / \int_{I} \frac{d x}{W(x)}\right]^{1 / 2}
$$

so the left side of (2.10) must be bounded by $K+1$ for this value of $A$. This immediately gives condition $A_{2}$ with constant $(K+1)^{2} / 4$.

Lemma 5. If $1 \leq p<\infty, W(x)$ satisfies the condition $A_{p}$ then $\log W(x)$ is of bounded mean oscillation.

If $p=2$, Lemma 4 asserts that (2.8) is true. An application of Jensen's inequality to (2.8) then shows that

$$
\exp \frac{1}{|I|} \int_{I}|\log W(x)-\log A| d x \leq K
$$

for some $A>0$. Then $\log W(x)$ is of bounded mean oscillation by the definition. If $1 \leq p<2, W(x)$ satisfies $A_{2}$ by Hölder's inequality so the previous applies. If $2<p<\infty,[W(x)]^{-1 /(p-1)}$ satisfies condition $A_{p^{\prime}} ;$ this again implies that $\log W(x)$ is of bounded mean oscillation.

3. Periodic sufficiency results for $p>1$. This section will be devoted to proving the following theorems.

Theorem 4. If $1<p<\infty$ and $W(\theta)$ bas period $2 \pi$ and satisfies the $A_{p}$ condition, then there is a constant $C$, independent of $f$, such that

$$
\int_{-\pi}^{\pi}|\tilde{f}(\theta)|^{p} W(\theta) d \theta \leq C \int_{-\pi}^{\pi}|f(\theta)|^{p} W(\theta) d \theta
$$

for every function $f$ with period $2 \pi$.

Theorem 5. If $1<p<\infty$ and $w(\theta)$ bas period $2 \pi$ and satisfies the $A_{p}$ condition then there is a constant $C$, independent of $f$, such that

$$
\int_{-\pi}^{\pi}[T f(\theta)]^{k} W(\theta) d \theta \leq C \int_{-\pi}^{\pi}|f(\theta)|^{p} W(\theta) d \theta
$$

for every function $f$ with period $2 \pi$.

These theorems will be proved by establishing a series of lemmas. 
A function $W(\theta)$ will be said to satisfy the Helson-Szego condition if it is nonnegative, has period $2 \pi$ and there exist functions $U(\theta)$ and $V(\theta)$ of period $2 \pi$ such that $U(\theta)$ is bounded, $V(\theta)$ is bounded by a constant less than $\pi / 2$ and $W(\theta)=\exp [U(\theta)+\widetilde{V}(\theta)]$ for almost every $\theta$. The first lemma, Lemma 6 , is a Riesz type proof that the Helson-Szego condition is sufficient to insure that $W$ is a weight function for $L^{2}$. This is, of course, a consequence of the proof in [7]; it is also contained in [5]. The proof given here is a special case of the one given in [5]; it is included here since it is simpler than the proofs in either [5] or [7].

Lemma 6. If $W(\theta)$ satisfies the Helson-Szego condition, then there is a constant $C$, independent of $f$, such that for every $f(\theta)$ of period $2 \pi$,

$$
\int_{-\pi}^{\pi}|\tilde{f}(\theta)|^{2} W(\theta) d \theta \leq C \int_{-\pi}^{\pi}|f(\theta)|^{2} W(\theta) d \theta .
$$

Since $\exp U(\theta)$ is bounded above and also below away from zero, we may assume from the beginning that $W(\theta)=\exp \widetilde{V}(\theta)$. By [13, Vol. I, p. 254], $W(\theta)$ is integrable on $[-\pi, \pi]$. Let $W(z), V(z)$, and $\widetilde{V}(z), z=r e^{i \theta}$, denote the Poisson integrals of $W, V$, and $\widetilde{V}$ respectively. The function $w(z)=\exp [\widetilde{V}(z)-i V(z)]$ is analytic and satisfies $|w(z)| \leq W(z)$ by Jensen's inequality. Therefore, $w(z)$ belongs to the Hardy space $H^{1}$. Its boundary values $w\left(e^{i \theta}\right)$ are $W(\theta)[\cos V(\theta)-i \sin V(\theta)]$ and since $\|V\|_{\infty}<\pi / 2$, there is a positive constant $C$, depending only on $\|V\|_{\infty}$, such that $C W(\theta) \leq \operatorname{Re} w\left(e^{i \theta}\right) \leq w(\theta)$ and $C\left|w\left(e^{i \theta}\right)\right| \leq \operatorname{Re} w\left(e^{i \theta}\right) \leq\left|w\left(e^{i \theta}\right)\right|$.

Let $f(\theta)$ be any trigonometric polynomial and let $F(z)$ be the bounded analytic function which is the Poisson integral of $f(\theta)+\tilde{i f}(\theta)$. Since $[F(z)]^{2} w(z)$ is harmonic, we have for $0<r<1$,

$$
\frac{1}{2 \pi} \int_{-\pi}^{\pi}\left[F\left(r e^{i \theta}\right)\right]^{2} w\left(r e^{i \theta}\right) d \theta=[F(0)]^{2} w(0)
$$

Letting $r \rightarrow 1^{-}$, we obtain

$$
\frac{1}{2 \pi} \int_{-\pi}^{\pi}[f(\theta)+\tilde{i f}(\theta)]^{2} w\left(e^{i \theta}\right) d \theta=[F(0)]^{2} w(0) .
$$

Here $[F(0)]^{2}=\left[(1 / 2 \pi) \int_{-\pi}^{\pi} f(\theta) d \theta\right]^{2}$ is real and nonnegative and $\operatorname{Re} w(0)=$ $\cos \left[(1 / 2 \pi) \int_{-\pi}^{\pi} V(\theta) d \theta\right]$ is positive. Hence taking real parts in (3.1) we have

$$
\int_{-\pi}^{\pi}\left[\left([f(\theta)]^{2}-[\tilde{f}(\theta)]^{2}\right) \operatorname{Re} w\left(e^{i \theta}\right)-2 f(\theta) \widetilde{f}(\theta) \operatorname{Im} w\left(e^{i \theta}\right)\right] d \theta \geq 0
$$

or since all integrals are finite,

$$
\int_{-\pi}^{\pi}[\tilde{f}(\theta)]^{2} \operatorname{Re} w\left(e^{i \theta}\right) d \theta \leq \int_{-\pi}^{\pi}[f(\theta)]^{2} \operatorname{Re} w\left(e^{i \theta}\right) d \theta-2 \int_{-\pi}^{\pi} f(\theta) \widetilde{f}(\theta) \operatorname{Im} w\left(e^{i \theta}\right) d \theta .
$$

By our remarks above there is a positive constant $C$ such that

$$
\int_{-\pi}^{\pi}[\tilde{f}(\theta)]^{2} W(\theta) d \theta \leq C\left(\int_{-\pi}^{\pi}[f(\theta)]^{2} W(\theta) d \theta+\int_{-\pi}^{\pi}|f(\theta) \tilde{f}(\theta)| W(\theta) d \theta\right) .
$$


If we apply Schwarz's inequality to the second term on the right and denote

$$
A^{2}=\int_{-\pi}^{\pi}[\tilde{f}(\theta)]^{2} W(\theta) d \theta \text { and } B^{2}=\int_{-\pi}^{\pi}[f(\theta)]^{2} W(\theta) d \theta
$$

we obtain $A^{2} \leq C\left(B^{2}+A B\right)$ or $(A / B)^{2} \leq C[(A / B)+1]$. Therefore, there is a $C_{1}$, depending only on $C$, such that $A / B \leq C_{1}$. Since $W$ is integrable on $[-\pi, \pi]$, trigonometric polynomials are dense in the space of functions with norm $\left(\int_{-\pi}^{\pi}[f(\theta)]^{2} W(\theta) d \theta\right)^{1 / 2}<\infty$ and the lemma follows.

Lemma 7. Let $p$ satisfy $1<p<2$, let $W(\theta)$ satisfy the $A_{p}$ condition and the Helson-Szego condition. Then there is a constant $C$ independent of $f$ such that

$$
\int_{D_{a}} W(\theta) d \theta \leq C a^{-p} \int_{-\pi}^{\pi}|f(\theta)|^{p} W(\theta) d \theta
$$

for all $a>0$ and all functions $f$ with period $2 \pi$.

If the integral on the right side of the conclusion is infinite, the lemma is immediate. We may, therefore, exclude this case; Lemma 2 then shows that $f$ is integrable on $[-\pi, \pi]$ and $\tilde{f}(\theta)$ exists for almost every $\theta$.

Fix an $a>0$ and $f(\theta)$ for which the right side of the conclusion is finite. If $\int_{-\pi}^{\pi}|f(\theta)| d \theta \geq 2 \pi a$

$$
\int_{D_{a}} W(\theta) d \theta \leq \int_{-\pi}^{\pi} W(\theta) d \theta\left[\frac{1}{2 \pi a} \int_{-\pi}^{\pi}|f(\theta)| d \theta\right]^{p} .
$$

Hölder's inequality shows that the right side of this inequality is bounded by

$$
(2 \pi a)^{-p}\left(\int_{-\pi}^{\pi} W(\theta) d \theta\right)\left(\int_{-\pi}^{\pi}[W(\theta)]^{-1 /(p-1)} d \theta\right)^{p-1}\left(\int_{-\pi}^{\pi}|f(\theta)|^{p} W(\theta) d \theta\right),
$$

and a use of the $A_{p}$ condition shows that this has the desired bound.

If $\int_{-\pi}^{\pi}|f(\theta)| d \theta<2 \pi a$, the usual Calderon-Zygmund decomposition (Theorem 4, p. 17 of [11]) can be applied to $f(\theta)$ considered as a function defined only on $[-\pi, \pi]$. Using $[-\pi, \pi]$ as the initial interval, this will give a sequence of intervals $I_{k}$ with disjoint interiors such that $|f(\theta)|<a$ for almost every $\theta$ that is in $[-\pi, \pi]$ but not in $\bigcup_{I_{k}}$ and

$$
a<\frac{1}{\left|I_{k}\right|} \int_{I_{k}}|f(\theta)| d \theta \leq 2 a .
$$

Define $g(\theta)=f(\theta)$ for $\theta$ in $[-\pi, \pi]-\bigcup I_{k}, g(\theta)=\left(1 /\left|I_{k}\right|\right) \int_{I_{k}} f(\phi) d \phi$ for $\theta$ in $I_{k}$ and define it outside $[-\pi, \pi]$ so that it will have period $2 \pi$. Define $b(\theta)=$ $f(\theta)-g(\theta)$. Let $G_{a}$ be the subset of $[-\pi, \pi]$ where $|\tilde{g}(\theta)|>a / 2$ and let $B_{a}$ be the subset of $[-\pi, \pi]$ where $|\widetilde{b}(\theta)|>a / 2$. Then since $D_{a} \subset B_{a} \cup G_{a}$, it will be sufficient to show that

$$
\int_{B_{a}} W(\theta) d \theta \leq C a^{-p} \int_{-\pi}^{\pi}|f(\theta)|^{p} W(\theta) d \theta
$$


and

$$
\int_{G_{a}} W(\theta) d \theta \leq C a^{-p} \int_{-\pi}^{\pi}|f(\theta)|^{p} W(\theta) d \theta
$$

where the $C$ is independent of $f$.

To prove (3.3) first define $I_{k}^{*}$ to be the interval with the same center as $I_{k}$ but which is three times as long. Let $H_{k}$ be the union of $I_{k}^{*}$ with the two intervals obtained by translating $I_{k}^{*}$ by $2 \pi$ and let $A=[-\pi, \pi]-\bigcup_{H_{k}} \cdot A$ is defined this way so that the periodic extension of $A$ does not intersect any $I_{k}^{*}$. Then $\int_{B_{a}} W(\theta) d \theta$ is bounded by the sum of

$$
\sum_{k} \int_{H_{k}} W(\theta) d \theta
$$

and

$$
\frac{2}{a} \int_{A}|\tilde{b}(\theta)| W(\theta) d \theta
$$

Because of (3.2), (3.5) is bounded by

$$
\sum_{k}\left(\frac{1}{a\left|I_{k}\right|} \int_{I_{k}}|f(\theta)| d \theta\right)^{p} 3 \int_{I_{k}^{*}} W(\theta) d \theta .
$$

Using Hölder's inequality shows that this is bounded by 3 times

$$
\text { (3.8) } \sum_{k}\left(\frac{1}{a\left|I_{k}\right|}\right)^{p} \int_{I_{k}}|f(\theta)|^{p} W(\theta) d \theta\left(\int_{I_{k}}[W(\theta)]^{-1 /(p-1)} d \theta\right)^{p-1} \int_{I_{k}^{*}} W(\theta) d \theta \text {. }
$$

Now enlarging the interval of integration in the middle integral to $I_{k}^{*}$ and using the $A_{p}$ condition shows that (3.8) is bounded above by

$$
a^{-p} \sum_{k} C\left(\frac{\left|I_{k}^{*}\right|}{\left|I_{k}\right|}\right)^{p} \int_{I_{k}}|f(\theta)|^{p} W(\theta) d \theta
$$

Since $\left(\left|I_{k}^{*}\right| /\left|I_{k}\right|\right)=3$, this shows that (3.5) is bounded by the right side of (3.3) with a suitable $C$.

To show that (3.6) also has the desired bound, write it in the form

$$
\frac{2}{a} \int_{A} W(\theta)\left|\sum_{k} \int_{I_{k}} \frac{b(\phi) d \phi}{2 \tan (\theta-\phi) / 2}\right| d \theta \text {. }
$$

This is bounded by

$$
\frac{2}{a} \sum_{k} \int_{A} W(\theta)\left|\int_{I_{k}} \frac{b(\theta) d \theta}{2 \tan (\theta-\phi) / 2}\right| d \theta
$$

Now let $\phi_{k}$ be the center of $I_{k}$ and let $J_{k}$ be the complement of $I_{k}^{*}$ in $\left[-\pi+\phi_{k}\right.$, $\left.\pi+\phi_{k}\right]$. Because the inner integral in (3.10) is a periodic function of $\theta$ and $A \cap H_{k}$ is empty (3.10) is bounded above by 


$$
\frac{2}{a} \sum_{k} \int_{J_{k}} W(\theta)\left|\int_{I_{k}} \frac{b(\phi) d \phi}{2 \tan (\theta-\phi) / 2}\right| d \theta
$$

By its definition $\int_{I_{k}} b(\phi) d \phi=0$ so (3.11) equals

$$
\frac{2}{a} \sum_{k} \int_{J_{k}} W(\theta)\left|\int_{I_{k}} \frac{b(\phi)}{2 \tan (\theta-\phi) / 2}-\frac{b(\phi)}{2 \tan \left(\theta-\phi_{k}\right) / 2} d \phi\right| d \theta .
$$

Now $\left|I_{k}\right|<\pi$ so that $|\theta-\phi| \leq 3 \pi / 4$ and clearly $\left|\theta-\phi_{k}\right| / 2 \leq \pi / 2$. Therefore, there is a constant $C$, such that

$$
\left|\frac{1}{2 \tan (\theta-\phi) / 2}-\frac{1}{2 \tan \left(\theta-\phi_{k}\right) / 2}\right| \leq \frac{C\left|I_{k}\right|}{\left(\theta-\phi_{k}\right)^{2}} .
$$

Now taking the absolute value sign inside the inner integral in (3.12), interchanging the order of integration and using (3.13) then shows that (3.12) is bounded above by

$$
\frac{C}{a} \sum_{k} \int_{I_{k}}|b(\phi)|\left(\int_{J_{k}} \frac{W(\theta)\left|I_{k}\right| d \theta}{\left(\theta-\phi_{k}\right)^{2}}\right) d \phi .
$$

Now Lemma 3 shows that this is bounded by

$$
\frac{C}{a} \sum_{k}\left(\int_{I_{k}}|b(\phi)| d \phi\right)\left(\frac{1}{\left|I_{k}\right|} \int_{I_{k}} W(\theta) d \theta\right) .
$$

By its definition

$$
\int_{I_{k}}|b(\phi)| d \phi=\int_{I_{k}}\left|f(\phi)-\frac{1}{\left|I_{k}\right|} \int_{I_{k}} f(\theta) d \theta\right| d \phi \leq 2 \int_{I_{k}}|f(\phi)| d \phi .
$$

Substituting this into (3.14) shows that (3.14) is bounded above by

$$
\frac{C}{a} \sum_{k} \frac{1}{\left|I_{k}\right|}\left(\int_{I_{k}}|f(\phi)| d \phi\right)\left(\int_{I_{k}} W(\theta) d \theta\right) \text {. }
$$

Now (3.2) shows that (3.15) is bounded by

$$
C a^{-p} \sum_{k}\left(\frac{1}{\left|I_{k}\right|} \int_{I_{k}}|f(\phi)| d \phi\right)^{p}\left(\int_{I_{k}} W(\theta) d \theta\right) \text {. }
$$

This can now be shown to be bounded by the right side of (3.3) in the same way that this was shown for (3.7). This completes the proof of (3.3).

To prove (3.4), start with the fact that the left side of (3.4) is bounded by

$$
4 a^{-i} \int_{-\pi}^{\pi}|\tilde{g}(\theta)|^{2} W(\theta) d \theta
$$

Since $W$ satisfies the Helson-Szego condition, Lemma 6 shows that (3.16) is bounded by 


$$
C a^{-2} \int_{-\pi}^{\pi}|g(\theta)|^{2} W(\theta) d \theta
$$

By its definition $|g(\theta)| \leq 2 a$ for almost all $\theta$; therefore, since $p<2$ (3.17) is bounded by

$$
C a^{-p} \int_{-\pi}^{\pi}|g(\theta)|^{p} W(\theta) d \theta
$$

Now (3.18) can be written in the form

$$
\begin{aligned}
C a^{-p} \int_{[-\pi, \pi]-\cup I_{k}}|f(\theta)|^{p} W(\theta) d \theta \\
+C a^{-p} \sum_{k} \int_{i_{k}}\left[\frac{1}{\left|I_{k}\right|} \int_{I_{k}}|f(\phi)| d \phi\right]^{p} W(\theta) d \theta .
\end{aligned}
$$

The second term can now be estimated in the way that (3.7) was. This completes the proof of Lemma 7.

Lemma 8. Let $p$ satisfy $1<p<2$, let $W(\theta)$ satisfy the $A_{p}$ condition and the Helson-Szego condition. Then there is a constant $C$, independent of $f$, such that

$$
\int_{-\pi}^{\pi}|\tilde{f}(\theta)|^{p} W(\theta) d \theta \leq C \int_{-\pi}^{\pi}|f(\theta)|^{p} W(\theta) d \theta
$$

for every $f$ with period $2 \pi$.

By Lemma $A$ there is an $r$ satisfying $1<r<p$ such that $W(\theta)$ satisfies the condition $A_{r}$. By Lemma 7 there is a constant $C$, independent of $f$, such that for $a>0$,

$$
\int_{D_{a}} W(\theta) d \theta \leq C a^{-r} \int_{-\pi}^{\pi}|f(\theta)|^{r} W(\theta) d \theta
$$

By Lemma 6 there is also a constant $C$ such that for $a>0$,

$$
\int_{D_{a}} W(\theta) d \theta \leq C a^{-2} \int_{-\pi}^{\pi}|f(\theta)|^{2} W(\theta) d \theta
$$

The conclusion of Lemma 8 then follows immediately from the Marcinkiewicz interpolation theorem, [13, Vol. II, p. 112].

The following lemma from [4] will be needed.

Lemma C. Assume that $1<p<\infty$ and there is a $K$, independent of $f$, such. that

$$
\int_{-\pi}^{\pi}|\tilde{f}(\theta)|^{p} W(\theta) d \theta \leq K \int_{-\pi}^{\pi}|f(\theta)|^{p} W(\theta) d \theta
$$

for every $f$ with period $2 \pi$. Then there is a constant $C$ depending only on $p$ and K such that 


$$
\int_{-\pi}^{\pi}|\tilde{f}(\theta)|^{p^{\prime}} W(\theta)^{-1 /(p-1)} d \theta \leq C \int_{-\pi}^{\pi}|f(\theta)|^{p^{\prime}} W(\theta)^{-1 /(p-1)} d \theta
$$

where $f$ is any function of period $2 \pi$ and $1 / p+1 / p^{\prime}=1$.

This is Lemma 3, p. 376 of [4]; it is proved by a simple duality argument.

Lemma 9. Let $p$ satisfy $1<p \leq 2$ and let $W(\theta)$ satisfy the $A_{p}$ condition. Then there is a constant $C$, independent of $f$ and $a$, such that

$$
\int_{D_{a}} W(\theta) d \theta \leq C a^{-p} \int_{-\pi}^{\pi}|f(\theta)|^{p} W(\theta) d \theta
$$

for $a>0$ and functions $f$ with period $2 \pi$.

This lemma is the same as Lemma 7 except that the requirement that $W$ satisfy the Helson-Szego condition has been dropped. The proof of Lemma 7 can be repeated through the end of the proof of (3.3) since this part of the proof did not use the Helson-Szego condition. The proof of Lemma 9, therefore, can be completed by proving that (3.4) is true with the hypotheses of Lemma 9.

To do this apply Lemma 5 and Lemma $B$ to $W(\theta)$. This shows that there are bounded functions $U(\theta)$ and $V(\theta)$ with period $2 \pi$ such that $W(\theta)=\exp [U(\theta)+\widetilde{V}(\theta)]$ for almost every $\theta$. Choose an $r$ so that $r>2$ and $|V(\theta)| /(r-1)$ is bounded by $\pi / 4$. Then $[W(\theta)]^{-1 /(r-1)}$ satisfies the Helson-Szego condition. Furthermore, since $1 /(r-1)<1 /(p-1)$, Hölder's inequality shows that $[W(\theta)]^{-1 /(r-1)}$ satisfies the condition $A_{r^{\prime}}$. Since $1<r^{\prime}<2$, Lemma 8 can be applied to show that there is a constant $C$, independent of $b$, such that

$$
\int_{-\pi}^{\pi}|\tilde{b}(\theta)|^{r^{\prime}} W(\theta)^{-1 /(r-1)} d \theta \leq C \int_{-\pi}^{\pi}|b(\theta)|^{r^{\prime}} W(\theta)^{-1 /(r-1)} d \theta
$$

for every function $h(\theta)$ with period $2 \pi$. Lemma $C$ then implies the existence of a constant $C$, independent of $b$, such that

$$
\int_{-\pi}^{\pi}|\widetilde{b}(\theta)|^{r} W(\theta) d \theta \leq C \int_{-\pi}^{\pi}|b(\theta)|^{r} W(\theta) d \theta
$$

for every function $b(\theta)$ with period $2 \pi$.

Now to prove (3.4) start with the fact that the left side of (3.4) is bounded above by

$$
2^{r} a^{-r} \int_{-\pi}^{\pi}|\tilde{g}(\theta)|^{r} W(\theta) d \theta
$$

Applying (3.20) shows that (3.21) is bounded by

$$
C a^{-r} \int_{-\pi}^{\pi}|g(\theta)|^{r} W(\theta) d \theta
$$

Since $|g(\theta)| \leq 2 a$ for almost every $\theta,(3.22)$ is bounded by

$$
C a^{-p} \int_{-\pi}^{\pi}|g(\theta)|^{p} W(\theta) d \theta
$$


Since (3.23) is the same as (3.18), the proof that (3.23) is bounded by the right side of (3.4) is the same as the proof that (3.18) has that bound. This completes the proof of Lemma 9.

The proof of Theorem 4 for $1<p<2$ follows from Lemma 9 in the same way that Lemma 8 followed from Lemmas 7 and 6 . For $p>2$ the result follows from Lemma $C$ and the result for $1<p<2$. For $p=2$ use Lemma $A$ to obtain an $r$, $1<r<2$, such that $W$ satisfies the condition $A_{r}$. By the first case proved in this theorem, there is a $C$ such that

$$
\int_{-\pi}^{\pi}|\widetilde{f}(\theta)|^{r} W(\theta) d \theta \leq C \int_{+\pi}^{\pi}|f(\theta)|^{r} W(\theta) d \theta
$$

Since $W$ also satisfies the condition $A_{4}$, by the second case proved in this theorem there is a $C$ such that

$$
\int_{-\pi}^{\pi}|\widetilde{f}(\theta)|^{4} W(\theta) d \theta \leq C \int_{-\pi}^{\pi}|f(\theta)|^{4} W(\theta) d \theta
$$

The Marcinkiewicz interpolation theorem then implies that there is a $C$ such that

$$
\int_{-\pi}^{\pi}|\widetilde{f}(\theta)|^{2} W(\theta) d \theta \leq C \int_{-\pi}^{\pi}|f(\theta)|^{2} W(\theta) d \theta
$$

To prove Theorem 5 use will be made of the fact that

$$
T f(\theta) \leq C\left[f^{*}(\theta)+(\widetilde{f})^{*}(\theta)\right]
$$

where ${ }^{*}$ denotes the Hardy-Littlewood maximal function operator defined in (2.2). Inequality (3.24) can be proved by combining the proof of Theorem 7.38, p. 279, Vol. I of [13] with Lemma 7.1, p. 154, Vol. I of [13]. By Corollary 4 of [9] there is a constant $C$, independent of $g$, such that

$$
\int_{-\pi}^{\pi}\left[g^{*}(\theta)\right]^{p} W(\theta) d \theta \leq C \int_{-\pi}^{\pi}|g(\theta)|^{p} W(\theta) d \theta
$$

for every $g$ with period $2 \pi$ since $W$ satisfies the $A_{p}$ condition. Theorem 5 follows immediately by using (3.24) and (3.25) and then applying Theorem 4.

4. A weak type result for $p=1$.

Theorem 6. If $W(\theta)$ has period $2 \pi$ and satisfies the $A_{1}$ condition, $a>0$ and $f(\theta)$ bas period $2 \pi$, then there is a constant $C$, independent of $f$ and $a$, such that

$$
\int_{E_{a}} W(\theta) d \theta \leq C a^{-1} \int_{-\pi}^{\pi}|f(\theta)| W(\theta) d \theta
$$

To prove Theorem 6, follow the proof of Lemma 7 through the decomposition of $f(\theta)$ into the sum of the functions $b(\theta)$ and $g(\theta)$. Let $B_{a}$ be the subset of $[-\pi, \pi]$ where $T b(\theta)>a / 2$ and let $G_{a}$ be the subset of $[-\pi, \pi]$ where $T g(\theta)>a / 2$. It will be sufficient to prove that 


$$
\int_{B_{a}} W(\theta) d \theta \leq C a^{-1} \int_{-\pi}^{\pi}|f(\theta)| W(\theta) d \theta
$$

and

$$
\int_{G_{a}} W^{\prime}(\theta) d \theta \leq C a^{-1} \int_{-\pi}^{\pi}|f(\theta)| W(\theta) d \theta
$$

where the $C$ is independent of $f$ and $a$.

To prove (4.1) we will first prove that if $\theta \in A$, then

$$
\sup _{\epsilon>0}\left|\int_{\epsilon<|\theta-\phi| \leq \pi} \frac{b(\phi) d \phi}{2 \tan (\theta-\phi) / 2}\right| \leq 12 b^{*}(\theta)+\sum_{k}\left|\int_{I_{k}} \frac{b(\phi) d \phi}{2 \tan (\theta-\phi) / 2}\right|
$$

where $A$ is defined as in Lemma 7 and $b^{*}(\theta)$ is the maximal function of $b$ as defined in (2.2).

To prove (4.3) fix $\epsilon>0$ and let $Q$ be the union of $[\theta-\epsilon, \theta+\epsilon]$ and the two intervals obtained from this interval by translating by $2 \pi$. Let $R$ be the set of $k$ 's such that $Q$ intersects $I_{k}$ and let. $S$ be the set of the other $k$ 's. Because of the periodicity of the integrand

$$
\left|\int_{\epsilon \leq|\theta-\phi| \leq \pi} \frac{b(\phi) d \phi}{2 \tan (\theta-\phi) / 2}\right|=\left|\int_{[-\pi, \pi]-Q} \frac{b(\phi) d \phi}{2 \tan (\theta-\phi) / 2}\right|,
$$

and this is bounded by the sum of

$$
\sum_{k \in R}\left|\int_{I_{k}-Q} \frac{b(\phi) d \phi}{2 \tan (\theta-\phi) / 2}\right|
$$

and

$$
\sum_{k \in S}\left|\int_{I_{k}} \frac{b(\phi) d \phi}{2 \tan (\theta-\phi) / 2}\right|
$$

Now let $Q^{*}$ be the union of $[\theta-2 \epsilon, \theta+2 \epsilon]$ and its two translates by $2 \pi$. Given a $k$ in $R$, since $\theta$ is not in $H_{k}$ and $Q$ intersects $I_{k}, I_{k}$ is a subset of $Q^{*}$. Therefore, (4.5) is bounded by

$$
3 \int_{\epsilon<|\theta-\phi|<2 \epsilon}\left|\frac{b(\phi)}{\theta-\phi}\right| d \phi .
$$

It is immediate that (4.7) is bounded by $12 b^{*}(\theta)$. It is also immediate that (4.6) is bounded by the second term on the right side of (4.3); this completes the proof of (4.3).

Now let $B_{a 1}$ be $\bigcup_{H_{k}}$, let $B_{a 2}$ be the subset of $[-\pi, \pi]$ where $b^{*}(\theta)>a / 48$, and let $B_{a 3}$ be the subset of $A$ where 


$$
\sum_{k}\left|\int_{I_{k}} \frac{b(\phi) d \phi}{2 \tan \left(\theta-\phi_{k}\right) / 2}\right|>\frac{a}{4} .
$$

Then $B_{a} \subset B_{a 1} \cup B_{a_{2}} \cup B_{a_{3}}$ and the proof of (4.1) will consist of verifying (4.1) with $B_{a}$ replaced by each of the $B_{a i}$. To prove it for $B_{a 1}$ use (3.2) to show that

$$
\int_{B_{a 1}} W(\theta) d \theta \leq \sum_{k} \frac{3}{a\left|I_{k}\right|} \int_{I_{k}}|f(\theta)| d \theta \int_{I_{k}^{*}} W(\theta) d \theta .
$$

The right side of (4.8) is bounded by

$$
\sum \frac{3}{a\left|I_{k}\right|} \int_{I_{k}}|f(\theta)| W(\theta) d \theta\left[\begin{array}{c}
\operatorname{ess} \sup _{\theta \in I_{k}} \\
W(\theta)
\end{array}\right] \int_{I_{k}^{*}} W(\theta) d \theta .
$$

The $A_{1}$ condition shows that this has the desired bound.

The estimation for $B_{a 2}$ follows immediately from Theorem 1 of [9]. For $B_{a 3}$ start with the fact that

$$
\int_{B_{a 3}} W(\theta) d \theta \leq 4 a^{-1} \int_{A} W(\theta) \quad \sum_{k}\left|\int_{I_{k}} \frac{b(\phi) d \phi}{2 \tan (\theta-\phi) / 2}\right| d \theta .
$$

The procedure used to estimate (3.9) can now be repeated to show that (4.9) is bounded by (3.15). The procedure used to estimate (4.8) can then be repeated to complete this part. This completes the proof of (4.1).

To prove (4.2) start with the fact that

$$
\int_{G_{a}} W(\theta) d \theta \leq a^{-2} \int_{-\pi}^{\pi}[T g(\theta)]^{2} W(\theta) d \theta .
$$

By Hölder's inequality $W(\theta)$ satisfies the $A_{2}$ condition so Theorem 5 asserts that the right side of (4.10) is bounded above by

$$
C a^{-2} \int_{-\pi}^{\pi}|g(\theta)|^{2} W(\theta) d \theta
$$

and since $|g(\theta)| \leq 2 a$ for almost every $\theta$, (4.11) is bounded by

$$
C a^{-1} \int_{-\pi}^{\pi}|g(\theta)| W(\theta) d \theta
$$

The fact that this is bounded by the right side of (4.2) follows in the same way that it was shown that (3.18) was bounded by the right side of (3.4).

5. Necessity for the periodic case.

Theorem 7. Let $W(\theta)$ be nonnegative and have period $2 \pi$ and assume that $1 \leq p<\infty$ and that there is a $C$, independent of $a$ and $f$, such that

$$
\int_{D_{a}} W(\theta) d \theta \leq C a^{-p} \int_{-\pi}^{\pi}|f(\theta)|^{p} W(\theta) d \theta
$$


where $f(\theta)$ is a function of period $2 \pi$ and $a>0$. Then $W(\theta)$ satisfies the $A_{p}$ condition or $W(\theta)=0$ for almost all $\theta$ or $W(\theta)=\infty$ for almost all $\theta$.

Fix an interval $I$ with $0 \leq|I| \leq \pi / 4$. We will show that

$$
\left(\int_{I} W(\theta) d \theta\right)\left(\int_{I}[W(\theta)]^{-1 /(p-1)} d \theta\right)^{p-1} \leq C|I|^{p}
$$

where $C$ is independent of $I$. This is sufficient to show that $W(\theta)$ satisfies one of the asserted conditions because of the periodicity of $W$.

Assume that $p>1$ and define $A=\int_{I}[W(\theta)]^{-1 /(p-1)} d \theta$. If $A=0,(5.2)$ is true because of the convention $0 \cdot \infty=0$.

If $A=\infty$, then $[W(\theta)]^{-1 / p}$ is not in $L^{p^{\prime}}$ on $I$. Therefore, there is a periodic function $g(\theta)$ that is 0 outside $I$ and its periodic images such that $g$ is in $L^{p}$ on $I$ and $\int_{I} W^{-1 / p} g d \theta=\infty$. Let $f(\theta)=[W(\theta)]^{-1 / p} g(\theta)$ and let $J$ be one of the two intervals with $|J|=|I|$ and exactly one end point in common with $I$. Then since $|\tilde{f}(\theta)|=\infty$ on $J, J \subset D_{a}$ for every $a$ and

$$
\int_{J} W(\theta) d \theta \leq C a^{-p} \int_{I}|f(\theta)|^{p} W(\theta) d \theta=C a^{-p} \int_{I}[g(\theta)]^{p} d \theta
$$

for every $a>0$. Therefore, $\int_{J} W d \theta=0$ and this implies that $\int_{J} W^{-1 /(p-1)} d \theta=\infty$. The argument can then be repeated to show that $\int_{I} W d \theta=0$ and this implies (5.2).

If $0<A<\infty$, let $f(\theta)=[W(\theta)]^{-1 /(p-1)}$ on $I$ and its periodic images and 0 elsewhere. For $\theta$ in $J$,

$$
|\tilde{f}(\theta)| \geq C \int_{I} \frac{f(\phi)}{|\theta-\phi|} d \phi \geq \frac{C A}{|I|} .
$$

Then let $a=C A /|I|$ in (5.1) and use the fact that $D_{a} \supset J$. This gives

$$
\int_{J} W(\theta) d \theta \leq C|I|^{p} A^{1-p}
$$

If $\int_{J} W^{-1 /(p-1)} d \theta=\infty$, the reasoning used for the case $A=\infty$ would produce the contradiction $A=\infty$. If $\int_{J} W^{-1 /(p-1)} d \theta=0, W(\theta)=\infty$ almost everywhere in $J$; this contradicts (5.3). Therefore,

$$
0<\int_{J}[W(\theta)]^{-1 /(p-1)} d \theta<\infty .
$$

Then repeating the reasoning used to obtain (5.3) shows that

$$
\left[\int_{I} W(\theta) d \theta\right]\left[\int_{J}[W(\theta)]^{-1 /(p-1)} d \theta\right]^{p-1} \leq C|I|^{p} .
$$

Now multiply (5.3) by $A^{p-1}$ and multiply the resulting inequality by (5.5). This gives

$$
\left[\int_{J} W(\theta) d \theta\right]\left[\int_{J}[W(\theta)]^{-1 /(p-1)} d \theta\right]^{p-1}\left[\int_{I} W(\theta) d \theta\right] A^{p-1} \leq C|I|^{2 p} .
$$


Because of (5.3) and (5.4) $0<W(\theta)<\infty$ for almost every $\theta$ in $J$. Therefore, by Hölder's inequality the product of the first two terms in (5.6) is bounded below by $|I|^{p}$. This proves (5.2) in this case.

If $p=1$ and ess $\inf _{\theta \in I} W(\theta)=\infty$, then (5.2) is true for all $C$. Otherwise, given $\epsilon>0$, there is a subset $E$ of $I$ such that $|E|>0$ and $W(\theta) \leq \epsilon+$ ess $\inf _{\phi \in I} W(\phi)$ for all $\theta$ in $E$. Define $f(\theta)=1$ on $E$ and its periodic images and 0 elsewhere. Then $|\tilde{f}(\theta)| \geq C|E| /|I|$ on $J$ and (5.1) implies that

$$
\int_{J} W(\theta) d \theta \leq \frac{C|I|}{|E|} \int_{E} W(\theta) d \theta \leq C|I|[\epsilon+\underset{\phi \epsilon I}{\operatorname{ess} \inf } W(\phi)] .
$$

Since this is true for every $\epsilon>0$, it follows that

$$
\int_{J} W(\theta) d \theta \leq C|I| \text { ess } \inf W(\phi) .
$$

The reasoning that led from (5.3) to (5.2) can now be used to show that (5.7) implies (5.2).

6. Weighted mean convergence of Fourier series. In this section $s_{n}(f, \theta)$ will be used to denote the $n$th partial sum of $f$ 's Fourier series. To avoid complications, $s_{n}(f, \theta)$ will be assigned the value $\infty$ if $f$ is not integrable. The result to be proved is the following.

Theorem 8. If $W(\theta)$ is nonnegative and has period $2 \pi$ and $1<p<\infty$, then the following statements are equivalent.

(a) There is a constant $C$, independent of $I$, such that for every interval $I$,

$$
\left[\frac{1}{|I|} \int_{I} W(\theta) d \theta\right]\left[\frac{1}{|I|} \int_{I}[W(\theta)]^{-1 /(p-1)} d \theta\right]^{p-1} \leq C
$$

(b) There is a constant $C$, independent of $f$, such that for every $f$ of period $2 \pi$

$$
\int_{-\pi}^{\pi}\left|s_{n}(f, \theta)\right|^{p} W(\theta) d \theta \leq C \int_{-\pi}^{\pi}\left|f^{\prime}(\theta)\right|^{p_{W}} W(\theta) d \theta .
$$

(c) If $f(\theta)$ bas period $2 \pi$ and $\int_{-\pi}^{\pi}|f(\theta)|^{p} W(\theta) d \theta<\infty$, then

$$
\lim _{n \rightarrow \infty} \int_{-\pi}^{\pi}\left|f(\theta)-s_{n}(f, \theta)\right|^{p} W(\theta) d \theta=0
$$

The proof that (a) implies (b) and (b) implies (c) is essentially the proof given for Theorem 6.4, p. 266, Vol. I of [13]. With the notation $g_{n}(\theta)=f(\theta) \cos n \theta$ and $b_{n}(\theta)=f(\theta) \sin n \theta$ and $s_{n}^{*}(f, \theta)=\tilde{g}_{n}(\theta) \sin n \theta-\widetilde{b}_{n}(\theta) \cos n \theta$,

$$
\left|s_{n}^{*}(f, \theta)-s_{n}(f, \theta)\right| \leq \frac{1}{2 \pi} \int_{-\pi}^{\pi}|f(\phi)| d \phi .
$$

Then 


$$
\left|s_{n}(f, \theta)\right| \leq \frac{1}{2 \pi} \int_{-\pi}^{\pi}|f(\phi)| d \phi+\left|\tilde{g}_{n}(\theta)\right|+\left|\tilde{h}_{n}(\theta)\right| .
$$

Hölder's inequality and the condition in statement (a) can be used on the first term and Theorem 1 can be used on the second and third terms to show that statement (a) implies statement (b).

If $W(\theta)=\infty$ almost everywhere then statement (c) is trivial. If $W(\theta)<\infty$ on a set of positive measure, then statement (b) implies that $\int_{-\pi}^{\pi} W(\theta) d \theta<\infty$, and, therefore, trigonometric polynomials are dense in the set of functions with norm $\left(\int_{-\pi}^{\pi}|f(\theta)|^{p} W(\theta) d \theta\right)^{1 / p}<\infty$. Statement (c) then follows from statement (b) in the same way that this implication is shown in the proof of Theorem 6.4, Vol. I, p. 266 of [13].

To prove that statement (c) implies statement (b), observe first that if $W(\theta)=0$ almost everywhere or $W(\theta)=\infty$ almost everywhere, then statement (b) is true. To prove that (c) implies (b) for other $W$ 's it will first be shown that statement (c) implies that $W^{-1 /(p-1)}$ and $W$ are integrable on $[-\pi, \pi]$.

To show that $W^{-1 /(p-1)}$ is integrable on $[-\pi, \pi]$ if statement (c) holds and $W(\theta)>0$ on a set of positive measure, assume the contrary. Then $W^{-1 / p}$ is not in $L^{p^{\prime}}$ on $[-\pi, \pi]$. Therefore, there is a $g$ in $L^{p}$ on $[-\pi, \pi]$ such that $g W^{-1 / p}$ is not in $L^{1}$ on $[-\pi, \pi]$. Let $f=g W^{-1 / p}$. Then $f$ satisfies the first part of statement (c) but not the second since the fact that $f$ is not in $L^{1}$ implies that for every $n\left|s_{n}(f, \theta)\right|=\infty$ for almost every $\theta$. This contradiction proves that $W^{-1 /(p-1)}$ is integrable on $[-\pi, \pi]$.

To prove that $W$ is integrable on $[-\pi, \pi]$ if statement (c) holds and $W(\theta)<\infty$ on a set of positive measure, a simple duality argument suffices. It is easy to see that if statement (c) is true and $g^{p^{\prime}} W^{-1 /(p-1)}$ is integrable on $[-\pi, \pi]$, then

$$
\lim _{n \rightarrow \infty} \int_{-\pi}^{\pi}\left|s_{n}(g, \theta)-g(\theta)\right|^{p^{\prime}} W(\theta)^{-1 /(p-1)} d \theta=0 .
$$

The proof in the last paragraph then shows that $W(\theta)^{-1 /(p-1)}$ raised to the power $-1 /\left(p^{\prime}-1\right)$ is integrable on $[-\pi, \pi]$. This power of $W$ is equal to $W$.

Now since $\left|s_{n}(f, \theta)\right| \leq(n+1) \int_{-\pi}^{\pi}|f(\phi)| d \phi$ Hölder's inequality and the two integrability facts just proved show that for each $n$ the mapping from $f$ to $s_{n}(f)$ is a bounded operator in the space with norm $\left(\int_{-\pi}^{\pi}|f|^{p} W d \theta\right)^{1 / p}$. The BanachSteinhaus theorem can then be applied to statement (c) to get statement (b).

There remains only the proof that statement (b) implies statement (a). To do this fix an $I$ with $|I| \leq \pi / 4$ and let $n$ be the greatest integer less than or equal to $\pi / 4|l|$. Let $f(\theta)$ be nonnegative on $I$ and its periodic images and 0 elsewhere. Then for $\theta$ in $I$,

$$
\left|s_{n}(f, \theta)\right|=\left|\frac{1}{\pi} \int_{I} \frac{f(\phi) \sin (n+1 / 2)(\theta-\phi)}{2 \sin (\theta-\phi) / 2} d \phi\right| \geq \frac{1}{\pi} \int_{I} \frac{|f(\phi)| \sin 3 \pi / 8}{\pi / 4 n} d \phi .
$$


Using this in statement (b) shows that for any nonnegative $f$,

$$
\left[\int_{I}|f(\phi)| d \phi\right]^{p}\left[\int_{I} W(\phi) d \phi\right] \leq C|I|^{p} \int_{I}|f(\phi)|^{p} W(\phi) d \phi .
$$

Let $A=\int_{I}[W(\phi)]^{-1 /(p-1)} d \phi$. If $A=0,(6.1)$ is immediate. If $0<A<\infty$, let $f(\phi)=[W(\phi)]^{-1 /(p-1)}$ in (6.2); this leads to (6.1) after dividing by $|I|^{p} A$. If $A=\infty, W(\phi)^{-1 / p}$ is not in $L^{p^{\prime}}$ on $l$. Then there is a $g(\phi)$ such that $g(\phi)$ is in $L^{p}$ on $I$ and $g(\phi) W(\phi)^{-1 / p}$ is not in $L^{1}$ on $l$. Let $f(\phi)=g(\phi)[W(\phi)]^{-1 / p}$, so $f(\phi)$ is not in $L^{1}$ on $I$. Using this $f$ in (6.2) shows that $\int_{I} W(\phi) d \phi=0$ and (6.1) follows in this case also. This completes the proof of Theorem 8.

7. Hilbert transform results. In this section we will use the following notation:

$$
\begin{aligned}
& H f(x)=\lim _{\epsilon \rightarrow 0^{+}} \frac{1}{\pi} \int_{|x-y|>\epsilon} \frac{f(y)}{x-y} d y, \\
& S f(x)=\sup _{\epsilon>0}\left|\frac{1}{\pi} \int_{|x-y|>\epsilon} \frac{f(y)}{x-y} d y\right|
\end{aligned}
$$

and

$$
K f(x)=\lim _{\epsilon \rightarrow 0^{+}} \frac{1}{\pi} \int_{|x-y|>\epsilon} f(y)\left[\frac{1}{x-y}+\frac{k(y)}{y}\right] d y
$$

where $k(y)-1$ for $|y| \geq 1$ and is 0 for $|y|<1$. As before, to avoid problems with one pathological case $H f$ and $S f$ will be defined to be $+\infty$ when $f(x) /(1+|x|)$ is not integrable.

'Theorem 9. If $1<p<\infty$ and $W(x)$ is nonnegative', then the following statements are equivalent; if $p: 1$, statements (a), (d), and (e) are equivalent.

(a) There is a constant $C$, independent of $I$, such that for every interval $I$.

$$
\left[\frac{1}{|I|} \int_{I} W(x) d x\right]\left[\frac{1}{|I|} \int_{I} W(x)^{-1 /(p-1)} d x\right]^{p-1} \leq C .
$$

(b) There is a constant C, independent of /, sucb that

$$
\int_{-\infty}^{\infty}|H f(x)|^{p} W(x) d x \leq c \int_{-\infty}^{\infty}|f(x)|^{p} W(x) d x .
$$

(c) There is a constant C, independent of /, such that

$$
\int_{-\infty}^{\sim}|S /(x)|^{p} W(x) d x \leq c ; \int_{-\infty}^{\infty}|f(x)|^{p} W(x) d x .
$$

(d) There is a constant (, independent of / and a, such that for $a>0$,

$$
\int_{\{|H| \mid>a\}} W(x) d x \leq \operatorname{ca}^{-p} \int_{-\infty}^{\infty}|f(x)|^{p} W(x) d x .
$$

(e) There is a constant $C$, independent of / and $a$, sucb that for $a>0$, 


$$
\int_{\{S f>a\}} W(x) d x \leq C a^{-p} \int_{-\infty}^{\infty}|f(x)|^{p} W(x) d x
$$

As in the case of Theorem 1, the hardest part of the proof consists of showing that (a) implies (b). There are two possible approaches to this proof. One is to use Theorem 4. This requires the fact that there is a $C$ depending only on $p$ and the $A_{p}$ constant of $W$ for which the conclusion of Theorem 4 holds. This is true but the proof is long and quite technical. It is easier to prove the following version of Lemma 6.

Lemma 10. Let $W(x)$ satisfy the $A_{2}$ condition and assume that $W(x)=$ $\exp [U(x)+K V(x)]$ where $U$ and $V$ are bounded and $\|V\|_{\infty}<\pi / 2$. Then there is a constant $C$, independent of $f$, such that

$$
\int_{-\infty}^{\infty}|H f(x)|^{2} W(x) d x \leq C \int_{-\infty}^{\infty}|f(x)|^{2} W(x) d x .
$$

With Lemma 10 to replace Lemma 6 the rest of the proof of Theorem 9 is like the proof of Theorem 1 with some obvious modifications. Consequently, the only part of the proof of Theorem 9 that will be discussed here is the proof of Lemma 10. Since the proof of Lemma 10 is similar to that of Lemma 6, we will only sketch the main points.

As in Lemma 6, we may as sume that $W(x)=\exp K V(x)$. Let $W(x, t), V(x, t)$, and $K V(x, t)$ denote the Poisson integrals of $W, V$, and $K V$ respectively. By Lemma $3 W(x, t)$ exists and satisfies $W(x, t) \leq C t^{-1} \int_{|x-y|<t} W(y) d y$ since $W$ satisfies the $A_{2}$ condition. $K V(x, t)$ exists; this is stated in [2] and proved in [3]. By verifying the Cauchy-Riemann equations we see that $K V(x, t)-i V(x, t)$ is analytic for $t>0$, and therefore, the function $w(x+i t)=\exp [K V(x, t)-i V(x, t)]$ is analytic for $t>0$. Since $\|V\|_{\infty}<\pi / 2$, there is a positive constant $C$, such that

$$
C|w(x+i t)| \leq \operatorname{Re} w(x+i t) \leq|w(x+i t)|
$$

and, therefore, since $\lim _{t \rightarrow 0}|w(x+i t)|=W(x)$ almost everywhere, $C W(x) \leq$ Re $w(x) \leq W(x)$. Moreover, since $|w(x+i t)| \leq W(x, t)$ by Jensen's inequality,

$$
|w(x+i t)| \leq c t^{-1} \int_{|x-y|<t} W(y) d y .
$$

Let $f(x)$ be any infinitely differentiable function with compact support and let

$$
F(z)=\frac{1}{i \pi} \int_{-\infty}^{\infty} \frac{f(y)}{y-z} d y, \quad z=x+i t
$$

$F$ is analytic in $t>0$ and is the Poisson integral of $f+i H f$. Since $f+i H f$ is bounded, $F$ is bounded and since $f$ has compact support, $|F(z)|=O\left(|z|^{-1}\right)$ as $|z| \rightarrow \infty$.

We claim the analytic function $F^{2} w$ is in $H^{1}$. For $t>0$, 


$$
\begin{aligned}
& \int_{-\infty}^{\infty}|F(x+i t)|^{2}|w(x+i t)| d x \\
& \quad \leq C \int_{-\infty}^{\infty} \frac{|w(x+i t)|}{1+x^{2}+t^{2}} d x \leq C \int_{-\infty}^{\infty} \frac{1}{t\left(1+x^{2}+t^{2}\right)} \int_{|x-y|<t} W(y) d y d x
\end{aligned}
$$

Changing the order of integration in this last expression and making a simple estimation of the size of the resulting inner integral, we obtain

$$
\int_{-\infty}^{\infty}|F(x+i t)|^{2}|w(x+i t)| d x \leq C \int_{-\infty}^{\infty} \frac{W(y)}{1+y^{2}} d y
$$

for $t>0$. By Lemma $3, F^{2} w$ is in $H^{1}$.

If $T_{R}, R>0$, denotes the isosceles triangle whose base is the interval $(-R, R)$ of the $x$-axis and whose altitude is $R$, it then follows from Cauchy's theorem that

$$
\int_{\partial T_{R}}[F(z)]^{2} w(z) d z=0 .
$$

The part of $\int_{\partial T_{R}} F^{2} w d z$ extended over the side of $T_{R}$ in the first quadrant is in absolute value at most a constant independent of $R$ times

$$
R^{-2} \int_{0}^{R}|w(x+i(R-x))| d x
$$

Replacing $w(x+i R-i x)$ by its upper bound

$$
\frac{C}{R-x} \int_{|x-y|<(R-x) / 2} W(y) d y
$$

and changing the order of integration we obtain at most a constant times

$$
R^{-2} \int_{-R}^{R} W(y)\left[\int_{2(R-y) / 3}^{2(R-y)} \frac{d x}{x}\right] d y \leq C R^{-2} \int_{-R}^{R} W(y) d y .
$$

The last expression tends to zero as $R \rightarrow \infty$ since $\int_{-\infty}^{\infty}\left[W(y) /\left(1+y^{2}\right)\right] d y<\infty$. Since the same argument can be used for the side of $T_{R}$ in the second quadrant we obtain from (7.4) the fact that

$$
\int_{-\infty}^{\infty}[f(x)+i H f(x)]^{2} w(x) d x=0 .
$$

The rest of the proof of Lemma 10 for functions which are infinitely differentiable with compact support is the same as the proof in Lemma 6 for trigonometric polynomials. Since $W$ is integrable on any compact set, infinitely differentiable functions with compact support are dense in the space of all $f$ with norm $\left(\int_{-\infty}^{\infty} f^{2} W d x\right)^{1 / 2}<\infty$. The lemma follows immediately from the se two facts.

8. The discrete case. Given a sequence $b_{n},-\infty<n<\infty$, define

$$
\tilde{b}_{n}=\sum_{n \neq m} \frac{b_{m}}{n-m} \text { and } T b_{n}=\sup _{k>0}\left|\sum_{|n-m| \geq k} \frac{b_{m}}{n-m}\right|
$$


$\widetilde{b}_{n}$ and $T b_{n}$ will be taken as $\infty$ if the defining sums do not converge. The analogue of Theorem 9 is then true and can be stated as follows.

Theorem 10. If $1<p<\infty$ and $w_{k} \geq 0$, then the following statements are equivalent; if $p=1$ statements (a), (d), and (e) are equivalent.

(a) There is a $C$ such that for every pair of integers $m$, nuth $m \leq n$,

$$
\left[\sum_{k=m}^{n} w_{k}\right]\left[\sum_{k=m}^{n}\left(w_{k}\right)^{-1 /(p-1)}\right]^{p-1} \leq C(n-m+1)^{p} .
$$

(b) There is a constant $C$, such that for every sequence $b_{k}, \sum_{k=-\infty}^{\infty}\left|\widetilde{b}_{k}\right|^{p} w_{k}$ $\leq C \Sigma_{k=-\infty}^{\infty}\left|b_{k}\right|^{p} w_{k}$.

(c) There is a constant $C$, sucb that for every sequence $b_{k}, \Sigma_{k=-\infty}\left|T b_{k}\right|^{p} w_{k}$ $\leq C \Sigma_{k=-\infty}^{\infty}\left|b_{k}\right|^{p} w_{k}$.

(d) There is a constant $C$, such that for every sequence $b_{k}$ and $a>0$, $\Sigma_{\left|\widetilde{b}_{k}\right|>a} w_{k} \leq C a^{-p} \Sigma_{k=-\infty}^{\infty}\left|b_{k}\right|^{p} w_{k}$.

(e) There is a constant $C$, such that for every sequence $b_{k}$ and $a>0$, $\Sigma_{T b_{k}>a} w_{k} \leq C a^{-p} \Sigma_{k=-\infty}^{\infty}\left|b_{k}\right|^{p} w_{k}$.

It is sufficient to prove that (a) implies (c) for $p>1$, (a) implies (e) for $p=1$ and (d) implies (a) for $p \geq 1$. The proof that (d) implies (a) is the same as the proof of Theorem 7. The proof that (a) implies (e) for $p=1$ will be omitted since it is like the proof that (a) implies (c) for $p>1$. Therefore, all that will be proved here is that (a) implies (c) for $p>1$.

To do this, fix sequences $b_{k}$ and $w_{k}$, define $W(x)$ to be $w_{k}$ for $k-1 / 4 \leq$ $x \leq k+1 / 4$ and linear between, and define $f(x)$ to be $b_{k}$ for $k-1 / 4 \leq x \leq k+1 / 4$ and 0 between. Then it is routine to verify that $W(x)$ satisfies the condition in part (a) of Theorem 9 . It is also simple to show that

$$
\left|\int_{|x-y|>n+1 / 2} \frac{2 f(y)}{x-y} d y-\sum_{|k-j|>n} \frac{a_{k}}{j-k}\right| \leq \int_{1 / 2<|x-y|} \frac{2|f(y)|}{(x-y)^{2}} d y
$$

provided that $k-1 / 4 \leq x \leq k+1 / 4$. It follows immediately that if $k-1 / 4 \leq x \leq k+1 / 4$, then

$$
T b_{k} \leq S f(x)+2 \int_{1 / 2 \leq|x-y|} \frac{|f(y)|}{(x-y)^{2}} d y .
$$

Now either by integrating by parts or using Corollary 2, p. 233 of [10], it is clear that the second term on the right side of (9.1) is bounded by $4 f^{*}(x)$ where $f^{*}(x)$ is the Hardy-Littlewood maximal function as defined in (2.2). Therefore,

$$
\sum_{-\infty}^{\infty}\left|T b_{k}\right|^{p} w_{k} \leq \sum_{-\infty}^{\infty} 2 \int_{k-1 / 4}^{i+1 / 4}\left|S f(x)+4 f^{*}(x)\right|^{p} \|(x) d x
$$

The right-hand side of (8.2) is bounded by 


$$
2^{p+1} \int_{-\infty}^{\infty}|S f(x)|^{p} W(x) d x+2^{3 p+1} \int_{-\infty}^{\infty}\left|f^{*}(x)\right|^{p} W(x) d x .
$$

Now use Theorem 9 on the first term of (8.3) and Theorem 2 of [9] on the second term. This shows that (8.3) is bounded by

$$
C \int_{-\infty}^{\infty}|f(x)|^{p} W(x) d x
$$

The integral in (8.4) is equal to $1 / 2 \Sigma\left|b_{k}\right|^{p} w_{k}$; this completes the proof that (a) implies (c) in Theorem 10.

\section{REFERENCES}

1. A. Benedek and R. Panzone, Continuity properties of the Hilbert transform, J. Functional Analysis 7 (1971), 217-234.

2. C. Fefferman, Characterizations of bounded mean oscillation, Bull. Amer. Math. Soc. 77 (1971), 587-588.

3. C. Fefferman and E. M. Stein, $H^{p}$-spaces of several variables, Acta Math. 129 (1972), 137-193.

4. F. Forelli, The Marcel Ries $z$ theorem on conjugate functions, Trans. Amer. Math. Soc. 106 (1963), 369-390. MR $26 \# 5340$.

5. V. F. Gapoškin, A generalization of the theorem of M. Riesz on conjugate functions, Mat. Sb. 46 (88) (1958), 359-372. (Russian) MR $20 \# 6000$.

6. G. H. Hardy and J. E. Littlewood, Some more theorems concerning Fourier series and Fourier power series, Duke Math. J. 2 (1936), 354-382.

7. H. Helson and G. Szegö, A problem in prediction theory, Ann. Mat. Pura Appl. (4) 51 (1960), $107-138$. MR $22 \# 12343$.

8. F. John and L. Nirenberg, On functions of bounded mean oscillation, Comm. Pure Appl. Math. 14 (1961), 415-426. MR 24 \#A1348.

9. B. Muckenhoupt, Weighted norm inequalities for the Hardy maximal function, Trans. Amer. Math. Soc. 165 (1972), 207-226.

10. - Poisson integrals for Hermite and Laguerre expansions, Trans. Amer. Math. Soc. 139 (1969), 231-242. MR 40 \#3158.

11. E. M. Stein, Singular integrals and differentiability properties of functions, Princeton Univ. Press, Princeton, N. J., 1970.

12. H. Widom, Singular integral equations in $L^{p}$, Trans. Amer. Math. Soc. 97 (1960), 131-160. MR $22 \# 9830$.

13. A. Zygmund, Trigonometrical series, 2nd rev. ed., Cambridge Univ. Press, New York, 1959. MR 21 \#6498.

14. M. Rosenblum, Summability of Fourier series in $L^{p}(d)$, Trans. Amer. Math. Soc. 105 (1962), 32-42.

DEPARTMENT OF MATHEMATICS, PURDUE UNIVERSITY, WEST LAFAYETTE, INDIANA 47907 (Current address of R. Hunt)

DEPARTMENT OF MATHEMATICS, RUTGERS UNIVERSITY, NEW BRUNSWICK, NEW JERSEY 08903 (Current address of B. Muckenhoupt and R. Wheeden) 